\title{
Transfer of facilitation in the rat
}

\author{
T. L. DAVIDSON and ROBERT A. RESCORLA \\ University of Pennsylvania, Philadelphia, Pennsylvania
}

\begin{abstract}
In two experiments, rats solved two concurrent discrimination problems in which one stimulus (i.e., a facilitator) signaled the reinforcement of another stimulus (i.e., a target). Then a transfer test assessed the capacity of facilitators trained in one problem to promote responding to targets trained in the other. Experiment 1 found that a facilitator promoted as much responding to such a transfer target as to the target with which it was originally trained. Transfer was not obtained with a pseudofacilitator that was uninformative, in training, about the reinforcement of its target. Experiment 2 manipulated the stimulus modality of the targets and facilitators. Its results indicated that transfer performance was not due to generalization between training and transfer targets or facilitators. These results parallel those from comparable autoshaping paradigms with pigeons, and they agree with the view that facilitators promote responding by lowering the threshold for activation of the US representation.
\end{abstract}

Several authors have recently reported results from a Pavlovian paradigm in which one stimulus $(A)$ is nonreinforced when presented alone but reinforced when presented in the context of another stimulus (B). For instance, Ross and Holland (1981) have described an "occasionsetting" paradigm in which rats receive food following the presentation of a tone but only if that tone has been preceded by a diffuse light. Similarly, Rescorla (1985) has reported a "facilitation" procedure in which pigeons receive a keylight that is reinforced only when embedded in a longer diffuse auditory or visual stimulus. In addition, both Rescorla (1985) and Jenkins (1985) have described procedures in which one keylight is reinforced only when preceded by another keylight. All of these procedures result in greater responding to the BA compound than to either $\mathrm{B}$ or $\mathrm{A}$ alone.

One may give various interpretations of this $\mathrm{A}-, \mathrm{BA}+$ discrimination learning. One possibility is summation of relatively low levels of associative strength controlled by $B$ and A. Even though B and A command too little strength to evoke a substantial response by themselves, they may combine to yield a strong response. A second possibility is that the animal treats the combined presentation of $B$ and $\mathrm{A}$ as a stimulus event that is not simply composed of the separate B and A elements. For instance, several authors (e.g., Rescorla, 1973) have suggested that when $B$ and $A$ are presented together there is a third emergent stimulus, which has been termed the "unique cue." That unique cue might then have its own association with the unconditioned stimulus (US) and that association might be responsible for responding to the compound BA presen-

This work was supported by Grant BNS-8308176 from the National Science Foundation and Grant T32 MH 17168 from the National Institute of Mental Health. This work was conducted while the first author was a postdoctoral researcher with the Department of Psychology and the Institute of Neurological Sciences at the University of Pennsylvania. Requests for reprints should be addressed to T. L. Davidson, who is now at the Department of Psychology, Virginia Military Institute, Lexington, VA 24450. tation. A third possibility is that one stimulus (A) has an association with the US and the other stimulus (B) acts to modulate the ability of $\mathrm{A}$ to use its association. Only in the presence of $B$ might $A$ be able to activate the US representation. This might come about either because B acts on the particular A-US association (Holland, 1983) or because B generally promotes the activation of the US representation by any of its associates (Rescorla, 1985).

Both Holland (1983) and Rescorla (1985) have argued that neither the simple summation of subthreshold excitation to $\mathrm{B}$ and $\mathrm{A}$ nor the presence of a unique cue can account for the responding that they observed to a BA compound. Instead, they interpreted their results in terms of the third possibility. That claim is based partly on the form of the response that is observed to the compound. That form is like one obtained when $A$ is directly conditioned. In both the rat occasion-setting studies and the pigeon facilitation studies, the B and A stimuli used were such that they evoked different response forms when they were individually paired with the US. For instance, Holland (1983) has reported that when rats receive light-food pairings the light comes to evoke rearing and passive sitting in front of the magazine; on the other hand, tonefood pairings result in the tone's evoking an enhanced startle response and "headjerking." Similarly, in pigeons a diffuse auditory or visual stimulus paired with food evokes increased general activity, whereas a localized keylight paired with food evokes directed pecking. The finding of interest is that after an A-, BA+ procedure, the compound produces a response like that which a conditioned A stimulus would control.

That particular response topography suggests that responding is based on an A-US association, the action of which is modulated by the presence of B. Such a topography would not be anticipated by a simple summation of the strengths to B and A. Both Holland (1983) and Rescorla (1985) have reported that after B is simply paired with the US, it does not have the power to augment the 
A's normal response when B and A are jointly presented. Moreover, it is not clear why a unique cue interpretation would anticipate that the response observed to the BA compound would be especially like that evoked by $A$ rather than that evoked by $B$.

Another result that is relevant to choosing among these alternatives is the successful transfer to other targets of B's ability to promote responding. For instance, Rescorla (1985) reported that for pigeon subjects a diffuse facilitator trained with one keylight also promoted responding to other keylights. Transfer of that sort suggests a generality of action that is not consistent with an account either in terms of a unique BA cue or in terms of B's acting on the association between a particular $A$ and the US.

Although such transfer to a new target is substantial for facilitation paradigms in pigeons, there is little relevant evidence from occasion-setting paradigms in rats. The only available report is an unpublished failure to obtain transfer (Holland, 1983). For that reason, the present studies examined transfer across different targets in an occasion-setting paradigm with rat subjects.

\section{EXPERIMENT 1}

The intention of this experiment was to provide transfer data for a facilitation procedure in rat subjects that would parallel the data reported by Rescorla (1985) for pigeons. Rats were trained concurrently in two facilitation paradigms. In each, an auditory A stimulus was followed by food when preceded by a visual B stimulus, whereas neither A nor B was reinforced when it was presented alone. Then each auditory stimulus was tested alone and in the presence of each of the visual facilitators. The issue was whether rats, like pigeons, would transfer a diffuse visual facilitator to an auditory stimulus that was trained as a target for another facilitator.

To be certain that the particular training paradigm was required to establish $B$ as a facilitator, a comparison group was also run. That group also received two concurrent discriminations. One discrimination was the same as the facilitation treatment described above. The other discrimination, however, comprised a "pseudofacilitation" paradigm, in which the auditory stimulus (A) was reinforced whether or not the visual stimulus (B) was present. With that paradigm, the animal received the same number of BA+ trials, but B was uninformative about when A would be reinforced. With an analogous procedure, Rescorla (1985) had found that pigeons do not develop facilitation. Consequently, the B stimulus from this procedure would serve as a comparison against which the transfer power of facilitators could be assessed.

\section{Method}

Subjects and Apparatus. The subjects were 16 naive male Sprague-Dawley rats, about 100 days old at the start of the experiment. Food deprivation was used to maintain the rats at $80 \%$ of their normal body weights. They took water ad lib throughout the experiment.
The experimental chambers consisted of four identical $22.9 \times 20.3 \times 20.3 \mathrm{~cm}$ boxes. Each chamber had a recessed food magazine in the center of the front wall and a 6-W jeweled lamp above the magazine which provided constant chamber illumination. In addition, the food magazine itself was dimly illuminated by a neon glow tube. The floor of the chamber was composed of .48 $\mathrm{cm}$ stainless steel rods spaced $1.9 \mathrm{~cm}$ apart. The front and back walls were aluminum; the side walls were clear acrylic plastic. Each chamber was enclosed in a light- and sound-resistant shell equipped with a clear acrylic plastic door to allow observation of the rats. A 6-W houselight, which served as a flashing light (2-Hz) signal, was mounted on the inside wall of the shell, about $10 \mathrm{~cm}$ from the inside wall of the experimental chamber, about $5 \mathrm{~cm}$ above it, and approximately midway between its front and back walls. The houselight was mounted between two 3-in speakers, one of which served to deliver auditory stimuli. An additional light assembly, providing a steady light cue, was mounted parallel to and $5 \mathrm{~cm}$ below the outside wall of the experimental chamber. Each assembly consisted of a tubular, 14-cm-long, 40-W incandescent lamp contained within a prism-shaped, $20-\mathrm{cm}$-long housing that was opaque on two sides and translucent on the third side, which faced the chamber. When illuminated, each assembly appeared as an evenly lit $10 \times 20 \mathrm{~cm}$ patch of light. The sound of a ventilating fan attached to the side of the shell served to continuously mask extraneous noise.

The rats' behaviors were monitored on a video recording system. This system consisted of a Panasonic low-light camera Model WV-250A) mounted $2.4 \mathrm{~m}$ from the experimental chambers in order to include all four chambers in its field of view, a Panasonic videocassette recorder (Model NV-2125), and a 22-in. Panasonic monitor (Model TR-220M).

Procedure. The rats were assigned to two groups of 8 rats each, matched on ad-lib body weight, and each group was subdivided into two squads of 4 rats each. The rats in each squad were assigned to different experimental chambers and were run in their assigned chamber throughout the experiment. The squads were run in the same order each day.

Each rat was magazine trained automatically with food pellets (45-mg, P.J. Noyes Co.) delivered on a variable-time (VT) 1-min schedule. Magazine training terminated following the delivery of 10 reinforcements. Throughout the experiment, a reinforcement consisted of two food pellets, delivered one at a time with an interval of $.25 \mathrm{sec}$; the total duration of feeder operation was $.5 \mathrm{sec}$.

Facilitation training began on the day following magazine training. All rats in Group E (experimental) were concurrently trained on two discrimination problems of the form LT+, T-, L-. On LT+ serial compound trials, a visual facilitator (L) was presented for $15 \mathrm{sec}$. An auditory target stimulus (T) overlapped the final $5 \mathrm{sec}$ of the light and coterminated in reinforcement $(+)$. On $\mathrm{T}-$ trials, a 5-sec target was presented by itself without reinforcement. In addition, although presentations of $L$ alone were contained within each LT trial, further 15-sec L- trials were introduced to ensure low levels of responding to the facilitator alone.

For half the rats in Group E, one discrimination problem had a flashing houselight serve as $L$ and a $1500-H z$ tone serve as $T$. The steady light and a 6-Hz clicker served as $\mathrm{L}$ and $\mathrm{T}$ stimuli in the other discrimination problem. For the remaining rats in Group $E$, the identities of the two $\mathrm{L}$ stimuli were reversed.

The rats in Group C (control) were treated identically to those in Group E except that one discrimination problem was disrupted by reinforcing $\mathrm{T}$ both when it was presented alone and when it occurred during $\mathrm{L}$. That is, one discrimination problem was of the form $\mathrm{LT}+, \mathrm{T}+, \mathrm{L}-$. The clicker was $\mathrm{T}+$ for half the Group $\mathrm{C}$ rats, and the tone was $T+$ for the remaining half.

All rats received three presentations of each of the six trial types during each 1-h session. The intertrial interval (ITI) was variable around a mean of $3.33 \mathrm{~min}$. Trials were presented in three differ- 
ent quasi-random sequences, with each sequence used once every 3 days. All rats received one training session a day for 24 days.

Testing began the day after the completion of training. During testing, two of each of the trial types administered during training continued to be given. In addition, each animal received two nonreinforced presentations of each of the two novel compounds consisting of the $\mathrm{L}$ and $\mathrm{T}$ from different training discriminations. The mean ITI remained $3.33 \mathrm{~min}$. All rats were given one test session a day for 3 consecutive days.

The behavior of each subject on each training and test trial was videotaped. Learning and transfer were assessed via a behavioral observation technique like that employed by Ross and Holland (1981). Three observations were made from the videotape for each rat during each 15 -sec trial period. This trial period began with the onset of the facilitator on serial compound and facilitator-alone trials and began $10 \mathrm{sec}$ before stimulus onset on target-alone trials. Paced by a once-per-1.25-sec auditory signal recorded on the tape, the observer shifted his gaze from chamber to chamber, observing each of the 4 rats once every $5 \mathrm{sec}$ during the trial period. Only one behavior was recorded on each observation.

The five categories of behavior recorded were similar to those described by Holland (1977). These were: (1) headjerking-short rapid horizontal and/or vertical movements of the head, usually oriented toward the food magazine; (2) rearing-standing on the hind legs with both forepaws off the grid floor. This category excludes grooming movements performed while the rat was standing on its hind legs; (3) magazine-standing motionless in front of the food magazine, with the nose or head close to or in the magazine; (4) locomotion-movement of all four feet in a forward motion; and (5) other-all behaviors other than those described above.

To assess the reliability of the scoring technique, a second observer scored selected sessions from each phase of the experiment. The two observers agreed on $92 \%$ of 288 joint observations.

\section{Results and Discussion}

Consistent with the results of other studies that have observed behavior elicited by food-reinforced auditory stimuli (e.g., Holland, 1977), headjerking emerged as the primary index of conditioning. Figure 1 depicts the mean percentage of observations scored as headjerking during the last $5 \mathrm{sec}$ of each trial (i.e., the target period) for Group E. The data are presented in blocks of four training sessions, collapsed across the two different types of $\mathrm{L}$ and $\mathrm{T}$ stimuli. During acquisition, Group $\mathrm{E}$ came to respond more rapidly during the LT compound than during either $\mathrm{L}$ or $\mathrm{T}$ alone. By the final block of training sessions, headjerking was reliably more frequent during LT than during either $\mathrm{T}$ alone or $\mathrm{L}$ alone [Wilcoxon $T \mathrm{~s}(8)$ $=0, p s<.01]$.

Headjerking for Group $\mathrm{C}$ is shown over the course of conditioning in Figure 2. Performance is shown separately for the stimuli that received facilitation (subscripted with a 1) and pseudofacilitation (subscripted with a 2) training procedures. The results from the facilitation procedure were similar to those for Group E. Responding to the $L_{1} T_{1}$ compound was greater than that to either the $L_{1}$ or $T_{1}$ elements when they were presented alone. By the final block of sessions, these differences were reliable $[T \mathrm{~s}(8)=0$, $p s<.01]$. By contrast, the results from the pseudofacilitation problem were quite different. For those stimuli, responding was high during both the $L_{2} T_{2}$ compound and the separately reinforced $\mathrm{T}_{2}$ element. Headjerking remained at a low level in the separately presented and non-

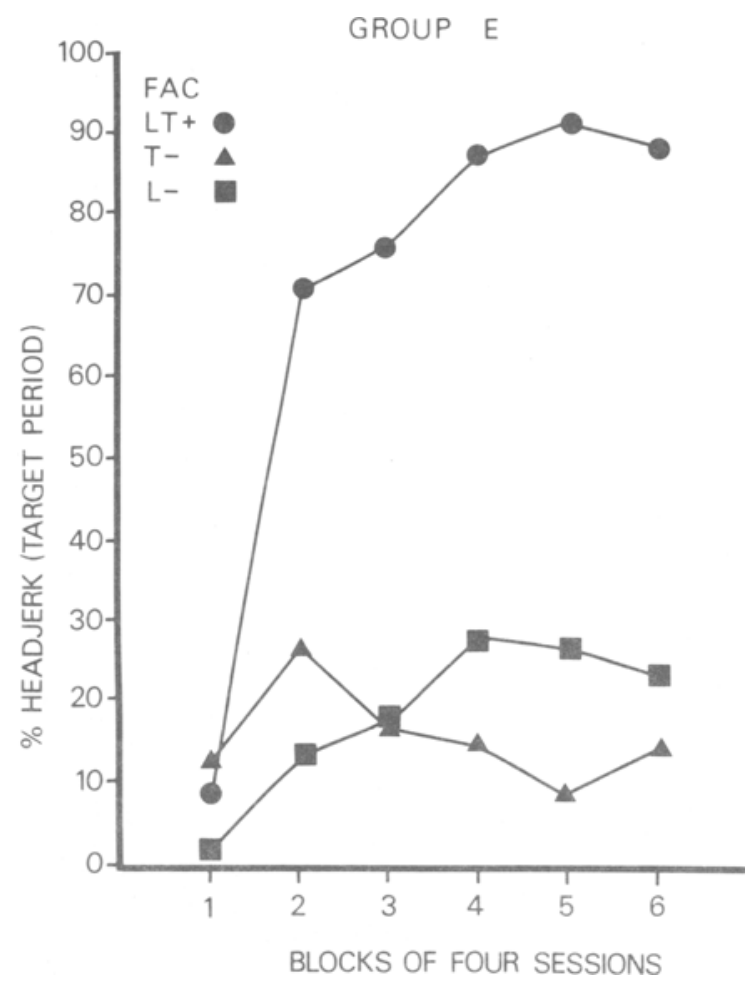

Figure 1. Mean percentage of target-period headjerking during acquisition for Group E of Experiment 1. Responding is shown during the reinforced serial compound (LT+) and the separate presentation of each of its elements ( $T-$ and $\left.L_{-}\right)$.

reinforced $\mathrm{L}_{2}$ trials. By the end of training, headjerking was reliably greater on $L_{2} T_{2}$ and $T_{2}$ trials than on $L_{2}$ trials $[T \mathrm{~s}(8)=0, p \mathrm{~s}<.01]$. However, the difference between responding on $\mathrm{L}_{2} \mathrm{~T}_{2}$ and $\mathrm{T}_{2}$ trials was not significant $[T(8)$ $=10]$.

These results show that both groups learned to behave appropriately under the facilitation paradigm. However, differential responding to the compound in Group $\mathrm{C}$ depended upon the differential reinforcement in the facilitation paradigm; it was not present in the pseudofacilitation procedure.

The results of the test session are shown in Figure 3. The top portion of that figure displays the percentage of headjerking in Group $E$ to each of the auditory stimuli $\left(T_{1}\right.$ and $T_{2}$ ) when they were presented alone and in conjunction with both their original (same subscript) and their transfer (different subscript) facilitator. For Group E, all stimuli, regardless of subscript, were trained within a facilitation procedure. For both auditory stimuli, responding continued to be greater during the original compounds $\left(\mathrm{L}_{1} \mathrm{~T}_{1}\right.$ and $\left.\mathrm{L}_{2} \mathrm{~T}_{2}\right)$ than during the targets alone $[T \mathrm{~s}(8)=$ $0, p s<.01]$. This indicates maintenance of the original discriminations during testing. More interestingly, responding was also greater during the transfer compounds $\left(L_{2} T_{1}\right.$ and $\left.L_{1} T_{2}\right)$ than during the $T_{1}$ and $T_{2}$ targets alone $[T \mathrm{~s}(8)=0, p \mathrm{~s}<.01]$. This indicates successful transfer of a facilitator to another target in Group E. The 


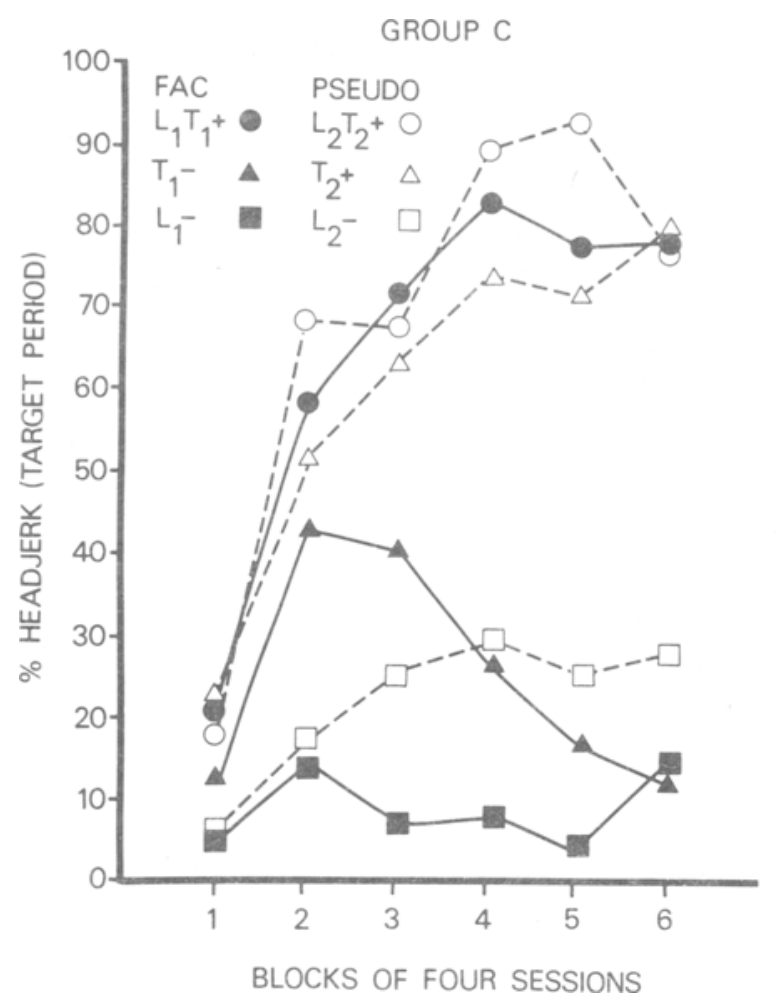

Figure 2. Mean percentage of target-period headjerking during acquisition for Group $\mathbf{C}$ of Experiment 1. Responding is shown to the serial compound $\left(L_{1} T_{1}+\right)$ and each element $\left(T_{1}-\right.$ and $\left.L_{1}-\right)$ during facilitation training, and to the serial compound $\left(L_{2} T_{2}+\right)$ and its elements $\left(T_{2}+\right.$ and $\left.\mathbf{L}_{2}-\right)$ trained in a pseudofacilitation paradigm.

difference in responding to the original and transfer compounds was not reliable $[T \mathrm{~s}(8)=6.5$ and 14.5$]$ with either target. In addition, headjerking was $16.7 \%$ on both $\mathrm{L}_{1}$ alone and $\mathrm{L}_{2}$-alone trials (not shown in Figure 3). This amount was significantly less than that observed during respective $L_{1} T_{2}$ and $L_{2} T_{1}$ transfer trials $[T \mathrm{~s}(8)=0$, ps $<.01]$.

The bottom portion of Figure 3 shows the results of testing for Group $\mathrm{C}$. The left-hand panel shows the results for the auditory stimulus trained in facilitation paradigm $\left(T_{1}\right)$, when it was presented alone, in compound with its training facilitator $\left(\mathrm{L}_{1}\right)$, and in compound with a pseudofacilitator $\left(L_{2}\right)$. Although the original facilitator, $L_{1}$, continued to elevate headjerking to $T_{1}$, the pseudofacilitator, $L_{2}$, did not. Headjerking on $L_{2} T_{1}$ transfer trials was not reliably different from that on $\mathrm{T}_{1}$-alone or on $\mathrm{L}_{2}$-alone trials $[T s(8)=7.5]$, but was reliably less than that on $\mathrm{L}_{1} \mathrm{~T}_{1}$ compound trials $[T(8)=0, p<.01]$. Headjerking on $\mathrm{L}_{2}$-alone trials was $8.3 \%$ (not shown in Figure 3 ). Furthermore, the difference in headjerking to the transfer compound between Groups $\mathbf{E}$ and $\mathbf{C}$ was also highly reliable [Mann-Whitney $U(8,8)=.5, p<.01]$. The results for the $T_{2}$ (the auditory stimulus trained in a pseudofacilitation paradigm) are shown in the bottom right-hand panel of Figure 3. Responding to that stimulus was high and nondifferential regardless of its manner of presentation.
These results suggest that substantial transfer is obtained when a facilitator is presented in conjunction with a target stimulus trained in another facilitation paradigm. That outcome implies a generality in the action of facilitators that is unexpected by accounts that emphasize either a unique cue or an action on a specific target-US association. Rather, they suggest that the facilitator generally enhances the ability of the US representation to be activated. Furthermore, these results do not support an account of responding to the $B A$ compound in terms of summation of the associative strengths of the $B$ and $A$ elements. It is always difficult to rule out such an account in the absence of specified rules for combining associative strengths of different stimuli. However, Groups $E$ and C showed substantial differences in responding to the $L_{2} T_{1}$ transfer compound despite displaying highly similar response rates to the $L_{2}$ and $T_{1}$ components when they
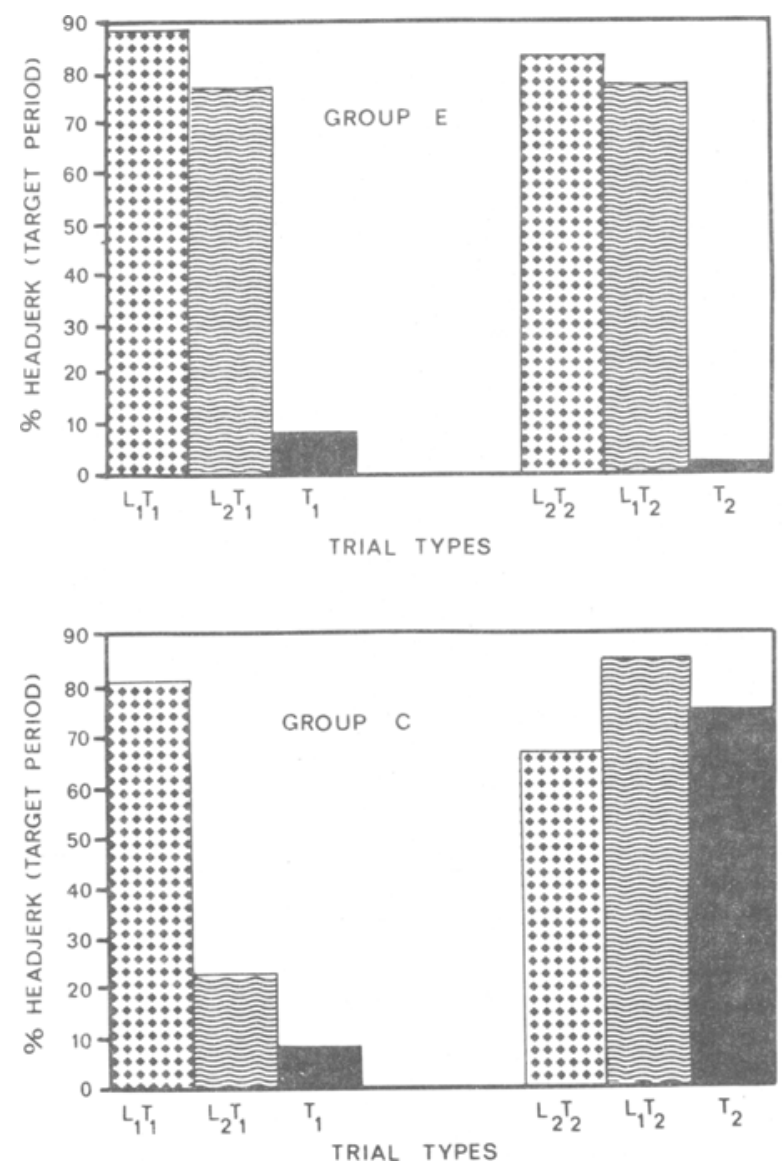

Figure 3. Mean percentage of target-period headjerking during testing in Experiment 1. The top panels show responding in Group $\mathbf{E}$ to each auditory stimulus $\left(T_{1}\right.$ and $\left.T_{2}\right)$ alone and in conjunction with their original (same subscript) and their transfer (difierent subscript) facilitators. The bottom left panel shows responding in Group $\mathbf{C}$ to the auditory stimulus given facilitation training $\left(T_{1}\right)$ when presented alone, when presented with its training facilitator $\left(L_{1} T_{1}\right)$, and when presented with its pseudofacilitator $\left(L_{2} T_{1}\right)$. Responding to the auditory stimulus $\left(T_{2}\right)$ trained in the pseudofacilitation paradigm is presented in the bottom right panel. 
were presented alone. Such a difference in responding to the compound in the face of similarities in responding to the elements alone challenges a broad range of combination rules.

These results also suggest that the ability of a stimulus to augment responding to a target depends upon the particular training procedure it is given. Simple reinforcement of $T_{2}$ in the presence of $L_{2}$ did not make $L_{2}$ a facilitator for Group C. Despite the high level of responding during the $L_{2} T_{2}$ compound in training, the separate reinforcement of $T_{2}$ when presented alone prevented $L_{2}$ from becoming a facilitator, as measured by its failure to promote responding to $T_{1}$. Apparently, in this procedure, as in autoshaping procedures with pigeons, a stimulus must be informative about the reinforcement of a target in order to become a facilitator.

\section{EXPERIMENT 2}

Interpretation of the results of Experiment 1 in terms of successful transfer from one target to another depends on the assumption that the organism is sensitive to the differences in identity of the targets and of the facilitators. Transfer would be neither surprising nor theoretically informative if the animal failed to identify $T_{1}$ as different from $T_{2}$ and $L_{1}$ as different from $L_{2}$. Because the two targets and the two facilitators lie in the same modality in Experiment 1, that alternative cannot be dismissed.

Experiment 2 attempted to reduce the plausibility of that alternative by using facilitation paradigms in which the two facilitators and the two targets were in different modalities. This was arranged in different ways for two groups. In Group Mixed, the animals were concurrently exposed to two facilitation paradigms. For one paradigm, the B stimulus was visual and the A was auditory; for the other paradigm, the B stimulus was auditory and the A was visual. Then the animals were tested with the novel facilitator/target compounds, which consisted of two stimuli both in the same modality. Group Same received the converse treatment. For that group, one facilitation paradigm had both $B$ and $A$ in the visual modality and a second had both in the auditory modality. Then transfer test compounds involved mixed modality presentations. For both groups, generalization between facilitators and between targets should be minimized by their differences in modality. Hence, should transfer of facilitation nevertheless occur, it would be much less likely to be based on failure to differentiate between training and transfer stimuli.

\footnotetext{
Method

Subjects and Apparatus. The subjects were 16 rats of the same description as those employed in Experiment 1; they were maintained as in Experiment 1. The apparatus was also the same as in Experiment 1 with following exceptions: (1) A 6-W jeweled lamp was located approximately $21 \mathrm{~cm}$ above the food magazine, $10 \mathrm{~cm}$ from the front wall of each conditioning chamber; this lamp replaced the 40-W incandescent light assembly used for the steady light in
}

Experiment 1. (2) Constant chamber illumination was provided by a red 6-W lamp mounted in the position of the houselight in Experiment 1 ; the $6-\mathrm{W}$ jeweled lamp above the magazine no longer provided constant chamber illumination, but replaced the houselight as a flashing light signal.

Procedure. The rats were assigned to groups, squads, and experimental chambers, and were magazine trained as in Experiment 1. Two groups were trained on two concurrent facilitation paradigms of the same form as those of Group $E$ in Experiment 1, except that stimuli comprising each problem were of the same modality for Group Same and of different modalities for Group Mixed. For Group Same, one discrimination problem was composed of only visual stimuli and the other was composed of only auditory cues. For half of the Group Same rats, a steady light facilitator and a flashing light target comprised one discrimination problem and a tone facilitator and a clicker target comprised the other. The identities of the respective facilitator and target stimuli were reversed within each discrimination problem for the remaining Group Same rats. For half of the Group Mixed rats, a tone was the facilitator and a flashing light was the target in one problem and a steady light and a clicker served as respective facilitator and target stimuli in the other. For the remaining Group Mixed rats, the identities of the respective facilitator and target stimuli were reversed within each discrimination problem. All rats were given 40 1-h training sessions. As in Experiment 1, each session was composed of three presentations of each of the six trial types comprising the two discrimination problems.

A transfer test assessed the capacity of facilitators trained in one discrimination problem to promote responding to targets trained in the other problem. Group Same, which was trained with samemodality facilitator and target stimuli, was tested with mixedmodality facilitator/target compounds. Group Mixed, which was trained with mixed-modality facilitator/target compounds, received test compounds of the same modality. Testing began the day after the completion of training. As in Experiment 1, both groups were given two nonreinforced test trials with both of their transfer facilitator-target compounds. Test trials were presented intermixed with normal training trials on each of 3 consecutive test days. Acquisition and transfer were assessed via the behavioral observation method described in Experiment 1.

\section{Results and Discussion}

As noted previously, Holland (1977) reported that the conditioned response to food-reinforced auditory cues took the form of headjerking. Furthermore, Holland also reported that rearing was the predominant form of the response to food-reinforced visual cues, although this outcome is not always obtained (e.g., Kaye \& Pearce, 1984). Since Experiment 2 employed stimuli from each of these modalities, it is appropriate to consider both headjerking and rearing as potential indices of discrimination learning.

Table 1 depicts mean percentage of headjerking and rearing to respective auditory and visual targets when they were presented in compound with their facilitator $(B A+)$

Table 1

Mean Percentage of Headjerking and Rearing as a Function of Target Modality

\begin{tabular}{|c|c|c|c|c|}
\hline \multirow{3}{*}{$\ldots$} & \multicolumn{3}{|c|}{ Function of Target Modality } & \\
\hline & \multicolumn{2}{|c|}{ Headjerk } & Rearing & \\
\hline & Auditory & Visual & Auditory & Visual \\
\hline $\begin{array}{l}\mathrm{BA}+ \\
\mathrm{A}-\end{array}$ & $\begin{array}{r}68.8 \\
4.2\end{array}$ & $\begin{array}{r}58.3 \\
0.0\end{array}$ & $\begin{array}{r}12.5 \\
8.3\end{array}$ & $\begin{array}{l}20.8 \\
25.0\end{array}$ \\
\hline
\end{tabular}

Note-Data represent observations made on target alone $(\bar{A}-)$ and facilitator/target $(\mathrm{BA}+)$ trials on the last day of training. 
and when they were presented alone $(\mathrm{A}-)$. These data are taken from the last day of training and are summed over groups. As shown in that table, the amount of headjerking on reinforced $B A+$ trials greatly exceeded that on A- target-alone trials within both stimulus modalities. These differences were highly reliable $[T \mathrm{~s}(16)<7$, $p s<.01]$. In contrast, Table 1 shows that rearing provided little indication of discrimination learning within either stimulus modality. Although rearing occurred slightly more on BA+trials than on A-trials with auditory target stimuli, this pattern was reversed when the targets were visual. In neither case were the differences reliable $[T s(16)>40]$. Hence, headjerking provided a clear index of discrimination learning with auditory and visual target stimuli, whereas rearing failed to do this for targets of either modality. Because of this, only headjerking will be discussed further as the measure of conditioning.

On the final day of training, both groups showed greater headjerking during the BA compound than during either the A or the comparable period of the B element. In Group Mixed, the percentages of headjerking were $56.3,0$, and 8.4, respectively, on BA, A, and B trials. The comparable figures for Group Same were 70.8, 4.2, and 16.7. Headjerking to facilitator/target compounds reliably exceeded that to targets and to facilitators alone for both groups $[T \mathrm{~s}(8)<2$, ps $<.02]$.

The results of the transfer test are presented in Figure 4. This figure depicts mean percentage of headjerking for both groups during their originally trained facilitator/target compound $\left(B_{1} A_{1}\right)$, their targets alone $\left(A_{1}\right)$, and their transfer facilitator/target compound $\left(B_{2} A_{1}\right)$, summed over the 3 test days. Discriminative performance was maintained by both groups during testing, at levels comparable to those found at the end of training. Headjerking for Group Mixed and for Group Same was significantly greater to

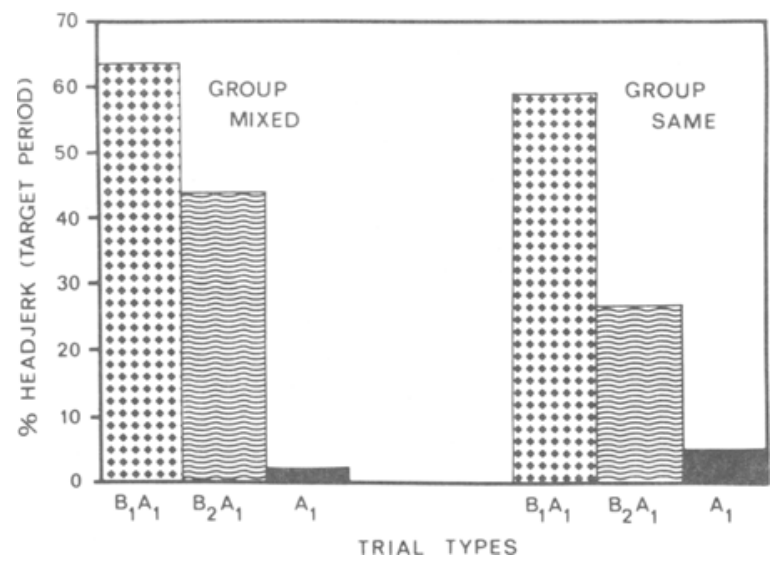

Figure 4. Mean percentage of target period headjerking during testing in Experiment 2. The data are stown for Group Mired (left) and Group Same (right) to their target stimull $\left(A_{1}\right)$ when presented alone and when presented in conjunction with their ortginn $\left(B_{1}\right)$ and their transfer $\left(B_{2}\right)$ facillitators. their originally trained facilitator/target compounds than to their targets $[T \mathrm{~s}(8)=1$ and $0, p s<.02$ and .01 , respectively].

More importantly, Figure 4 also indicates that both groups headjerked more to their transfer compounds $\left(B_{2} A_{1}\right)$ than they did to the separate presentations of the targets. This was especially clear for Group Mixed, which was trained with mixed-modality compounds and tested on same-modality compounds. In that group, responding was reliably greater to the transfer compound than to the target $[T(8)=0, p<.01]$ but not significantly different from that to the original training compound $[T(8)=5.5]$. Group Same showed similar results, with greater headjerking to the $B_{2} A_{1}$ compound than to $A_{1}$ alone $[T(8)=$ $0, p<.01]$. However, in this group, responding to the transfer compound $\left(\mathrm{B}_{2} \mathrm{~A}_{1}\right)$ fell reliably below that to the original training compound $\left(\mathrm{B}_{1} \mathrm{~A}_{1}\right)[T(8)=1, p<.02]$. The level of responding to the facilitators alone was at $10.4 \%$ and $14.6 \%$ in Groups Mixed and Same, respectively. For Group Mixed, that was reliably less than the level of responding to either the training or transfer compound $[T \mathrm{~s}(8)=1$ and $0, p \mathrm{~s}<.01]$. For Group Same, responding to the facilitator alone was reliably below that to the training compound $[T(8)=0, p<.01]$ but not that to the transfer compound $[T(8)=7]$.

These results confirm the transfer findings of Experiment 1 , under conditions that greatly reduce the plausibility of the hypothesis that transfer was due to failure to discriminate between targets or between facilitators.

\section{GENERAL DISCUSSION}

The results of these two experiments indicate an interchangeability of facilitators from one target to another. This interchangeability was not dependent upon the relative modalities of the training and transfer targets or the training and transfer facilitators. Consequently, that transfer is unlikely to be due to failure to discriminate between training and test targets or facilitators. Instead, the results indicate that a facilitator gains a response-promoting power that extends to targets different from that with which it was trained. These results from rats thus agree with those from comparable autoshaping paradigms with pigeons.

This successful transfer helps in making a choice among alternative interpretations of the responding to $\mathrm{BA}$ after $\mathrm{BA}+, \mathrm{A}-$ training. It makes less attractive interpretations in terms of an emergent stimulus that is unique to the BA compound or interpretations in terms of B's controlling a specific A-US association. Instead, it suggests a general effect of $B$ on various stimuli associated with the US. One interpretation is that B lowers the threshold for activation of the US representation, providing more ready access for any of its associates. That interpretation is complementary to one account of the conditioned inhibition that results from the converse $\mathrm{BA}-, \mathrm{A}+$ paradigm. The apparent response-inhibiting power acquired by $B$ in that 
paradigm has been described in terms of B's raising the threshold of activation of the US representation, thereby reducing access to the US of its excitatory associates. The parallel ability of a B so trained to transfer its inhibition to other stimuli associated with its US is consistent with that view (Rescorla \& Holland, 1977).

In contrast to our positive findings, Holland (1983) described a failure to find transfer in an unpublished experiment that used a BA+, A- feature-positive design with rats. Holland's experiment differed from those reported here in various ways. In the present procedure, the B stimulus preceded and overlapped the A stimulus, whereas Holland allowed $\mathbf{B}$ to terminate well before $\mathrm{A}$. In addition, Holland assessed transfer to a previously reinforced then extinguished stimulus rather than to a target within another discrimination problem. It may be that targets that have previously benefited from facilitation are more likely to allow transfer effects than are cues established as excitors under other conditions. Although Rescorla (1985) found successful transfer in the autoshaping preparation both with a $B$ bearing a trace relation to $A$ and with a trained and extinguished $A$ target, these variables may play a more important role in the present preparation. Finally, in the interest of guaranteeing low levels of responding to both the facilitator and the target, our procedure added separate nonreinforced presentations of $\mathrm{B}$ alone to the $\mathrm{A}-/ \mathrm{BA}+$ paradigm. It is possible that these additional B-trials in some way promoted B's ability to transfer to new targets. Holland (personal communication, 1985) has found some preliminary evidence supporting this possibility.

Another feature of our results that differs from that reported by Holland (1983) is the failure of visual targets to reliably evoke a conditioned response that differs from that evoked by auditory targets. Although Holland (1983) has reported substantial conditioned rearing to lights paired with food, our results find little evidence for conditioned rearing. Rather, our results seem more consistent with the view expressed by Kaye and Pearce (1984) that some instances of rearing to a light are orienting responses which represent more the attention the light controls than the conditioning that it gains. It seems likely that the detailed physical properties of the light stimulus may importantly affect the form of the response it produces (see Holland, 1977). However, the absence of clearly differentiable responses to auditory and visual stimuli clearly complicates the analysis of facilitation in this preparation.

Nevertheless, the present data suggest that transfer can be obtained across targets in this paradigm. That suggests a generality of action which encourages more theoretical analysis of the process of facilitation.

\section{REFERENCES}

Holland, P. C. (1977). Conditioned stimulus as a determinant of the form of the Pavlovian conditioned response. Journal of Experimen tal Psychology: Animal Behavior Processes, 3, 77-104.

Holland, P. C. (1983). Occasion-setting in Pavlovian feature positive discrimination. In M. L. Commons, R. J. Herrnstein, \& A. R. Wagner (Eds.), Quantitative analyses of behavior: Discrimination processes (Vol. 4, pp. 183-206). New York: Ballinger.

JENkINS, H. M. (1985). Conditioned inhibition of keypecking in the pigeon. In R. R. Miller \& N. E. Spear (Eds.), Information processing in animals: Conditioned inhibition (pp. 327-353). Hillsdale, NJ: Erlbaum.

KaYe, H., PEarce, J. M. (1984). The strength of the orienting response during Pavlovian conditioning. Journal of Experimental Psychology: Animal Behavior Processes, 10, 90-109.

RescorlA, R. A. (1973). Evidence for a unique-cue account of configural conditioning. Journal of Comparative \& Physiological Psychology, 85, 331-338.

ResCoRlA, R. A. (1985). Facilitation and inhibition. In R. R. Miller \& N. E. Spear (Eds.), Information processing in animals: Conditioned inhibition (pp. 299-326). Hillsdale, NJ: Erlbaum.

Rescorla, R. A., \& Holland, P. C. (1977). Associations in Pavlovian conditioned inhibition. Learning \& Motivation, 8, 429-447.

Ross, R. T., \& Holland, P. C. (1981). Conditioning of simultaneous and serial feature-positive discriminations. Animal Learning \& Behavior, 9, 293-303.

(Manuscript received December 5, 1985; revision accepted for publication July 8,1986 .) 